\title{
Agroecological aspects of application of Pseudomonas sp. DA1.2 in overcoming herbicidal stress in wheat
}

\author{
Maxim. D. Timergalin ${ }^{1,}$, Arina V. Feoktistova ${ }^{1}$, Timur V. Rameev ${ }^{1}$, Gaisar G. \\ Khudaygulov $^{1}$, Sergei N. Starikov ${ }^{1}$, and Sergei P. Chetverikov ${ }^{1}$ \\ ${ }^{1}$ Ufa Federal Research Centre of the Russian Academy of Sciences, Ufa Institute of biology, 450054 \\ Ufa, Russia
}

\begin{abstract}
This article submits results of laboratory and field experiments on the effect of an auxin-producing bacterial strain Pseudomonas sp. DA1.2 in comparison with Pseudomonas koreensis IB-4 on wheat plants in conjunction with the "Chistalan" herbicide treatment. Our work shows the positive effect of bacterial treatments on plant growth, the relative water content in leaves and the role of bacteria in the redistribution of $\mathrm{ABA}$ and IAA in wheat shoots under conditions of herbicidal stress. Application of Pseudomonas sp. DA1.2 together with the herbicide in the field of the steppe zone led to an increase in yield by $20 \%$ relative to the control variant. This bacterial strain helps to overcome herbicidal stress and is a promising agent for improving the technology of using synthetic auxins herbicides.
\end{abstract}

\section{Introduction}

Herbicides in controlling the weeds is an integral part of the modern agricultural system. Moreover, herbicides use leads to environmental pollution, and also causes stressful effects on cultivated plants, leading to their yield decrease [1].

Selective action of herbicides, i.e. their ability to inhibit growth of certain plant species and not affect other species, provides the possibility of their use in crop production. It is generally thought that 2,4-dichlorophenoxyacetic acid (2,4-D), one of the most famous herbicides that was first discovered and widely used, inhibits the growth of dicotyledonous weeds and does not affect the growth of monocotyledonous plants. This principle underlies in the use of 2,4-D in cereal crops (primarily wheat). Nevertheless, a generalization of the results of long-term tests of 2,4-D herbicide revealed a considerable number of cases of the negative effect of this herbicide on wheat growth [2].

One of the urgent tasks is the search for the effective anti-stress agents. Bacteria that stimulate plant growth (Plant Growth Promoting Bacteria - PGPB) can be considered as such promising agents. Their application helps to increase yield [3], and also improves condition of plants against the background of the herbicidal stress action [4]. The stimulating effect of bacteria is primarily associated with their ability to synthesize phytohormones (auxins, cytokinins, etc.) [5].

\footnotetext{
* Corresponding author: chelab007@yandex.ru
} 
The objective of our research was to assess the effect of a promising PGPB strain on growth and hormonal status of plants, as well as the prospect of its use as a biological antidote for herbicidal stress.

\section{Materials and methods}

The object of our research was soft spring wheat (Triticum aestivum L.) of the Kinelskaya Yubileinaya variety. The auxin-producing strain of Pseudomonas sp. Bacteria - DA 1.2, which is resistant to working concentrations of herbicides, with active substances based on 2,4-D, dicamba and others. The effect of the new isolate was compared with Pseudomonas koreensis IB-4 (a commercial biological product "Azolen"), which is a producer of auxins and cytokinins [6]. Both strains are stored in the collection of microorganisms at the Ufa Federal Research Centre of the Russian Academy of Sciences.

We used a complex preparation of selective action (against dicotyledons) as a herbicide, which is based on auxin-like active substances 2,4-D (2-ethylhexyl ether) and dicamba (sodium salt) - "Chistalan extra".

The experiment was carried out in laboratory and field conditions.

Wheat seeds were sterilized for 20 min with potassium permanganate solution and were germinated for 3 days in the laboratory. The seedlings were planted in 0.51 vessels filled with soil with a $10 \%$ sand content. Plants were grown on a light platform with a photon flux density PAR of $190 \mu \mathrm{mol} \cdot \mathrm{m}^{-2} \cdot \mathrm{s}^{-1}$ at a 14 -hour photoperiod and a temperature of $22-26{ }^{\circ} \mathrm{C}$. Soil moisture was maintained at a level in the range from $60 \%$ to $80 \%$ of the total soil water capacity.

7 days after seed germination, $1 \mathrm{ml}$ of an aqueous solution of "Chistalan" was applied to the soil and directly to plants by spraying (the working concentration of the herbicide was $0.5 \mathrm{ml} / \mathrm{l}$ ). A suspension of bacteria, grown in King B liquid medium, which was led to a titre of $108 \mathrm{CFU} / \mathrm{ml}$, was added to the herbicide tank mixture.

Level of phytohormones ABA and IAA was determined on the second day after the treatment of plants. Determination of hormone content was performed using enzyme-linked immunosorbent assay (ELISA) as described in [7-8].

A field experiment was conducted on the experimental plots of the steppe zone of the Trans-Urals in the summer of 2019. Treatment of crops was carried out at the stage of the third leaf appearance. 14 days after the treatments, the relative water content, weight and shoot length were determined in both experimental variants. The relative water content (RWC) was determined in the differentiated part of the wheat shoot leaf (1 leaf) according to the standard method. Yielding was evaluated in the field experiments.

\section{Results and discussion}

Although monocotyledonous plants are insensitive to the action of 2,4-D synthetic auxin herbicides, their use before the tillering stage can inhibit the wheat growth [2].

In our laboratory experiments, treatment with the "Chistalan" product inhibited the wheat growth, shoot's length (Fig. 1a) and its weight (Fig. 1b) compared with the control group of plants, and this showed herbicidal stress. 

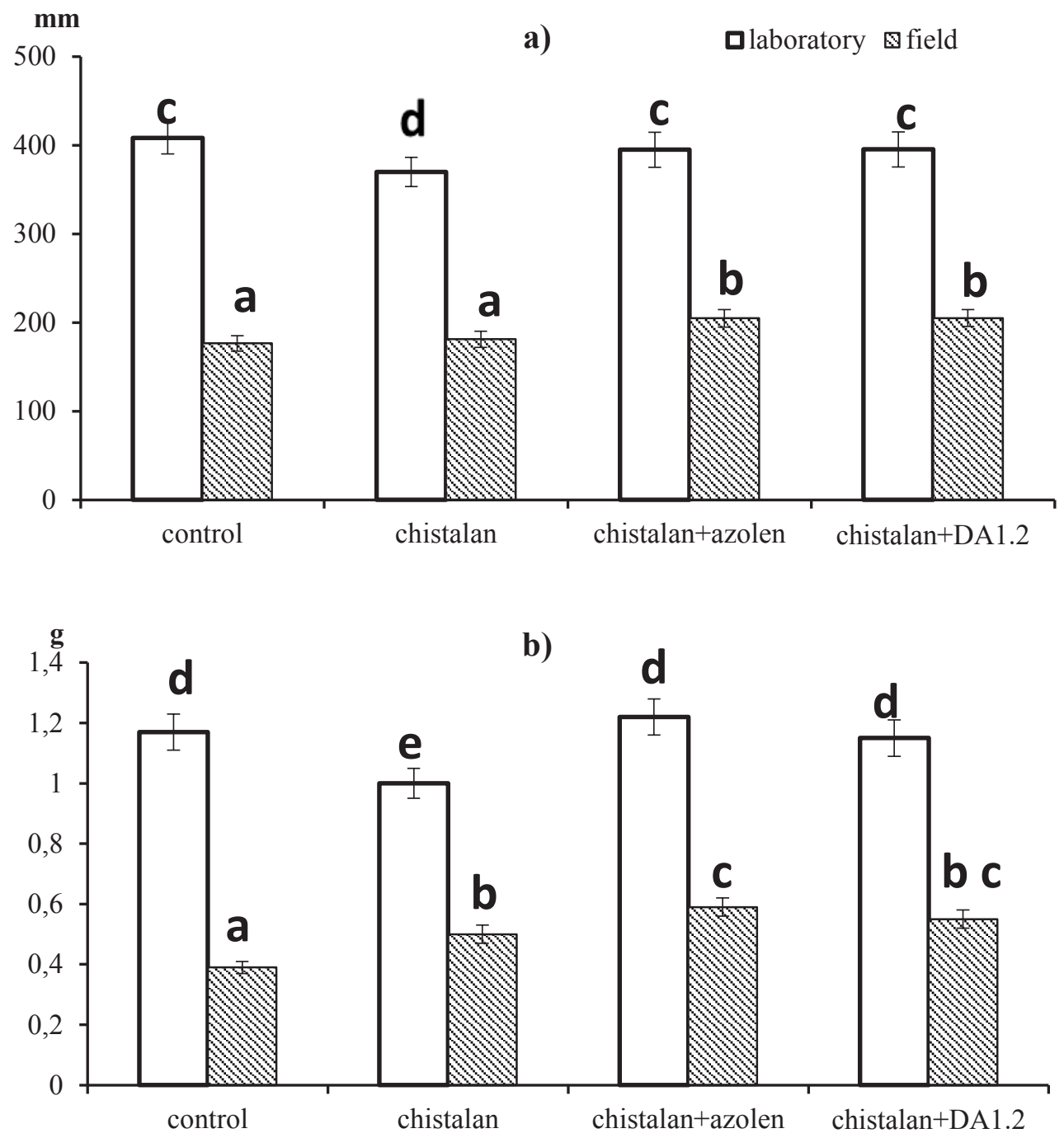

Fig. 1. Wheat shoot's length (a) and weight (b) in variants treated with the "Chistalan" herbicide, with addition of the "Azolen" product and the auxin-producing isolate DA1.2 into the tank mixture.

Statistically different values are marked with different letters, ANOVA, LSD test, $p \leq 0.05 ; n=6$.

It is generally thought that action of herbicides of the class of synthetic auxins on the sensitive plants is due to excessive accumulation of auxins in plants and a violation of their distribution between organs, thereby inhibiting plant growth [9].

The herbicide's effect was manifested in a three-fold increase in the content of auxins (Fig. 2a). Accumulation of auxins could be as the result of the absorption of exogenous auxins by plants, which, although they exhibit weak immunoreactivity with respect to IAA antibodies [7], are still able to react with antibodies at a high concentration of 2,4-D. An alternative explanation may be that the herbicide could affect the concentration of endogenous auxins. This explanation is consistent with the literature on the ability of 2,4-D to influence the IAA metabolism [10]. 

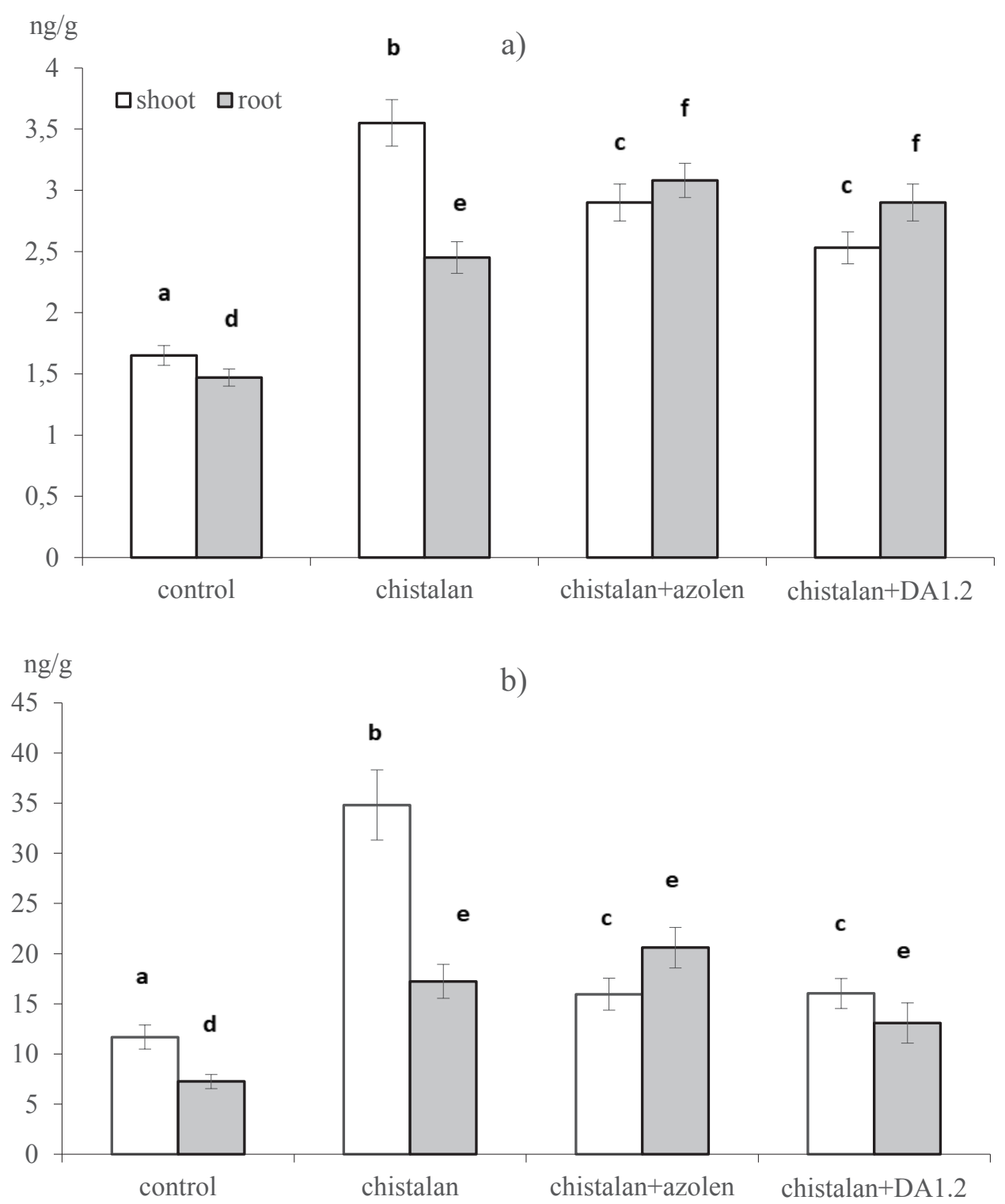

Fig. 2. Content of IAA (a) and ABA (b) in the shoots and roots (per g of wet weight) of Kinelskaya Yubileinaya wheat on the $2^{\text {nd }}$ day after treatment with the "Chistalan" herbicide; with the "Chistalan" herbicide, the "Azolen" product and auxin-producing strain DA1.2. Statistically different values are marked with different letters, ANOVA, LSD test, $\mathrm{p} \leq 0.05 ; \mathrm{n}=6$.

Treatment of plants with "Chistalan" was also accompanied by an increase in the concentration of ABA relative to the control variant by more than 1.5 times in the shoot and 2 times in the root. The reason for the ABA accumulation could be an increase in the level of auxins in plants, since it is well known that auxins contribute to the activation of ABA synthesis [11]. There is an opinion that the effect of 2,4-D on plants is precisely due to its ability to stimulate the ABA accumulation [12]. Since ABA is able to inhibit plant growth [13], an increase in its level in plants treated with "Chistalan" could be a reason for inhibiting the growth of wheat plants. 
At the same time, the shoot's weight significantly increased with the addition of "Azolen" relative to the variant with the herbicide alone (without bacteria) (by 15\%).

Adding of the bacterial compound "Azolen" to the herbicide product stimulated shoot growth; as a result, the plants in this experiment did not differ from the control variant (Fig. 1a).

A three-fold increase in the concentration of auxins in the shoot when treated only with a herbicide (without inoculating bacteria) was levelled under the influence of both bacteria strains, so that the level of auxins in the shoots exceeded the value of the untreated control variant by no more than 1.5 times. A decrease in the content of auxins could be due to the destruction of 2,4-D under the bacteria action. The ability of bacteria to destroy pesticides is known in the literature [14]. However, this assumption needs to be verified, given that the herbicide has been in contact with bacteria for a short time. An alternative explanation could be in normalizing metabolism of endogenous auxins under the influence of bacterial drugs.

One way or another, stabilization of the level of auxins in the variant with bacteria could favourably affect the ABA level. As can be seen from Figure 2b, co-treatment with bacteria and herbicides led to a decrease in the level of $\mathrm{ABA}$ accumulation in shoots compared to plants treated only with the herbicide.

Inhibition of wheat growth was not observed in the field, the shoot's length did not differ from the control variant, and the shoot's weight in plants treated with the herbicide was significantly higher. At the same time, we cannot talk about the complete absence of herbicidal stress in wheat, since under the conditions of a field experiment, plants from the control group could have a noticeable effect on the presence of weed vegetation and, as a result, water and/or nutrient deficiency.

Introduction of both bacterial strains of "Azolen" and DA1,2 in combination with the herbicide, stimulated shoot growth, as a result of which the shoot's length (Fig. 1a) and weight (Fig. 1b) were significantly higher than in the control variant and in plants treated only with "Chistalan".

An important feature of the bacteria action was their ability to maintain a high relative water content in plants (Table 1). The RWC was higher in the variants with bacterial treatments than in plants with the "Chistalan" background and in control variant. This trend was manifested both in the laboratory and in the field experiments.

An increase in the water level in the shoot could be due to an increase in its inflow from the roots. It is well known that the ABA hormone increases the water potential of plants not only due to changes in stomatal [15], but also in hydraulic conductivity [16]. Regulation of hydraulic conductivity under the influence of ABA most clearly manifests itself in the plants' roots, which corresponds to our data indicating that inoculation of bacteria against the background of the herbicide contributed to the redistribution of ABA in favour of the root.

Table 1. Relative water content (RWC) in plant leaves after treatment with the "Chistalan" herbicide; with the herbicide, "Azolen" and auxin-producing strain DA1.2. Average errors are indicated; $\mathrm{n}=9$.

\begin{tabular}{|c|c|c|}
\hline \multirow{2}{*}{ Treatment variant } & \multicolumn{2}{|c|}{ RWC, \% } \\
\cline { 2 - 3 } & Laboratory conditions & Field conditions \\
\hline control & $84.0 \pm 0.2$ & $82.5 \pm 1.2$ \\
\hline Chistalan & $81.8 \pm 1.1$ & $83.6 \pm 0.8$ \\
\hline Chistalan+Azolen & $91.8 \pm 1.6$ & $84.8 \pm 0.2$ \\
\hline Chistalan+DA1.2 & $91.7 \pm 2.0$ & $91.3 \pm 1.4$ \\
\hline
\end{tabular}

The yield in the control sample of plants was $18.6 \mathrm{c} / \mathrm{ha} ; 18.8 \mathrm{c} / \mathrm{ha}$ - when treated with "Chistalan"; 19.1 and $23.4 \mathrm{c} /$ ha - with addition of "Azolen" and DA1.2, respectively. 


\section{Conclusion}

Our data indicate that the auxin-producing strain of Pseudomonas sp. Bacteria - DA1.2, along with Pseudomonas koreensis IB-4 (“Azolen") helps to maintain water in leaf tissue under conditions of herbicidal stress, reduces its negative effect, which is expressed in violation of the redistribution of auxins and ABA.

At the same time, despite the fact that "Azolen" was effective in terms of influencing the wheat growth parameters under laboratory conditions, DA1.2 is more effective in the conditions of the arid climate of the Trans-Ural steppe and, when used together with the herbicide, increases yield up to $20 \%$ of the control level.

Thus, the considered bacterial strain exhibits the properties of an anti-stress agent of synthetic auxins, and can be recommended for optimizing the technology of herbicides application.

The study was carried out within the framework of the Ministry of education and science of Russia № 075-00326-19-00 on the topic № AAAA-A19-119021390081-1.

\section{References}

1. R. S. Kutuzova, N. I. Vorob'ev, Yu. V. Kruglov, Eurasian Soil Science 39, 195 (2006). DOI: $10.1134 / \mathrm{S} 1064229306020104$

2. S. Kumar, A. K. Singh, J. Chem. Pharm. Res. 2, 118 (2010)

3. J. K. Vessey, Plant and Soil 255, 571 (2003)

4. M. Ahemad, M. S. Khan, Pesticide Biochemistry and Physiology 98, 183 (2010). DOI: 10.1016/j.pestbp.2010.06.005

5. I. C. Dodd, N. Y. Zinovkina, V. I. Safronova, A. A. Belimov, Ann. Appl. Biol. 157, 361 (2010). DOI: 10.1111/j.1744-7348.2010.00439.x

6. G. F. Rafikova, T. Y. Korshunova, L. F. Minnebaev, S. P. Chetverikov, O. N. Loginov, Mikrobiologiya 85, 333 (2016)

7. S. U. Veselov, G. R. Kudoyarova, N. L. Egutkin, V. Z. Guili-Zade, A. R. Mustafina, E. M. Kof, Physiologia Plantarum 86, 93 (1992)

8. G. R. Kudoyarova, L. B. Vysotskaya, T. N. Arkhipova, L. Yu Kuzmina, N. F. Galimsyanova, L. V. Sidorova, I. M. Gabbasova, A. I. Melentiev, S. Yu. Veselov, Acta Physiologiae Plantarum 39 (2017). DOI: 10.1007/s11738-017-2556-9

9. K. Grossmann, Plant Signal Behav. 2, 421 (2007). DOI: 10.4161/psb.2.5.4417

10. Z. R. Sung, Planta 145, 339 (1979). DOI: 10.1007/BF00388358

11. H. Shuai, Y. Meng, X Luo, F. Chen, W. Zhou, Y. Dai, Y. Qi, J. Du, F. Yang, J. Liu, W. Yang, K. Shu, Sci. Rep. 7, 12620 (2017). DOI: 10.1038/s41598-017-13093-w

12. P. J. Christoffoleti, M. R. A. Figueiredo, L. E. P. Peres, S. Nissen, T. Gaines, Sci. Agric. 72, $356(2015)$

13. R. Lorrai, A. Boccaccini, V. Ruta, M. Possenti, P. Costantino, P. Vittorioso, AoB Plants 10 (2018). DOI: 10.1093/aobpla/ply061

14. L. Han, D. Zhao, C. Li, Brazilian Journal of Microbiology 46, 433 (2015). DOI: 10.1590/S1517-838246220140211

15. G. V. Sharipova, D. S. Veselov, G. R. Kudoyarova, M. D. Timergalin, S. Wilkinson, Russian Journal of Plant Physiology 59, 573 (2012). DOI: 10.1134/S1021443712040127 
16. G. R. Kudoyarova, D. S. Veselov, G. V. Sharipova, G. R. Akhiyarova, I. C. Dodd, S. Y. Veselov, Russian Journal of Plant Physiology 61, 188 (2014). DOI: 10.1134/S1021443714020071 